\title{
Ethnologies
}

\section{Chaire de recherche du Canada en patrimoine ethnologique}

\section{Laurier Turgeon, Célia Forget, Louise Saint-Pierre et Martin Fournier}

Volume 29, numéro 1-2, 2007

Retour à l'ethnographie

Back to Ethnography

URI : https://id.erudit.org/iderudit/018760ar

DOI : https://doi.org/10.7202/018760ar

Aller au sommaire du numéro

Éditeur(s)

Association Canadienne d'Ethnologie et de Folklore

ISSN

1481-5974 (imprimé)

1708-0401 (numérique)

Découvrir la revue

Citer cet article

Turgeon, L., Forget, C., Saint-Pierre, L. \& Fournier, M. (2007). Chaire de recherche du Canada en patrimoine ethnologique. Ethnologies, 29(1-2), 357-362. https://doi.org/10.7202/018760ar d'utilisation que vous pouvez consulter en ligne. 


\title{
Chaire de Recherche du Canada En Patrimoine ETHNOLOGIQUE
}

\author{
Laurier Turgeon \\ Université Laval \\ Laurier.Turgeon@hst.ulaval.ca
}

\section{L'Inventaire des ressources ethnologiques du patrimoine immatériel (IREPI)}

Activités de 2006

Les travaux d'inventorisation des ressources ethnologiques du patrimoine immatériel de la Chaire de recherche du Canada en patrimoine ethnologique se sont poursuivis au cours de la dernière année. Pendant l'été 2006, les étudiants-chercheurs sous la direction du titulaire de la Chaire ont sillonné les routes du Québec afin de recueillir les savoirs et les savoir-faire auprès des porteurs de traditions, des organismes, des entreprises. Ils ont également inventorié les espaces culturels traditionnels et les formes d'expression. Huit étudiants au baccalauréat et quatre étudiants à la maîtrise de l'Université Laval ont suivi une formation intensive qui portait à la fois sur les principes directeurs de l'IREPI, sur la méthodologie de recherche (enquête orale et observation directe effectuées dans une perspective de rechercheaction) et sur le rôle de l'enquêteur, tant en ce qui concerne le recensement des ressources ethnologiques que les actions culturelles. Les étudiants ont pu se familiariser avec le concept de patrimoine immatériel grâce à des ateliers offerts par des experts. Les étudiantschercheurs auront parcouru six régions du Québec dans 24 Municipalités régionales de comté (MRC) (Mauricie, Chaudière-Appalaches, Capitale-Nationale, Côte-Nord, Montérégie et Bas-Saint-Laurent) et auront réalisé près de 280 enquêtes orales auprès de différents individus et organismes. 
À l'automne 2006 débutaient le traitement et l'informatisation des données. Des guides d'écoute ont été produits pour l'indexation des enregistrements et la description sommaire de leur contenu, des fiches d'inventaire ont été complétées dans la banque de données IREPI, des photographies ont été traitées pour le web, des extraits audiovisuels ont été sélectionnés et intégrés au site Internet. Cinq étudiants ont été engagés aux trimestres d'automne 2006 et d'hiver 2007.

L'approche ethnologique privilégiée par la Chaire est la rechercheaction. Dans cette perspective, les étudiants ont consacré environ $30 \%$ de leur temps de travail aux actions culturelles. Conférences de presse, articles dans les journaux, participation à des émissions de radio et de télévision, avaient pour but de faire connaître et de mettre en valeur les ressources du patrimoine immatériel, ainsi que de redonner aux populations locales ce qu'elles avaient offert aux enquêteurs dans chacune des régions visitées. Grâce à la collaboration de nos partenaires régionaux, les étudiants ont pu bénéficier de soutiens pour l'organisation d'expositions et d'activités promotionnelles.

Quatre articles ont été publiés dans le journal d'information et d'opinion du Bas-Saint-Laurent et de la Gaspésie, Le Mouton Noir. À la suite du succès de ces publications, les étudiants ont été invités à publier quelques rubriques et photographies dans un guide touristique paru au printemps 2007 et un article dans la revue d'histoire de l'Université du Québec à Rimouski, L'Estuaire. En Montérégie, les étudiants ont publié des articles dans le Journal Saint-François et participé à l'émission de radio Train de vie. Les étudiants de la Côte-Nord ont participé à des émissions culturelles à la radio de Radio-Canada et l'équipe de Chaudière-Appalaches a rédigé plusieurs articles sur les porteurs de traditions de la Beauce pour le site Internet du Musée Marius Barbeau. Les articles et les émissions visaient à mettre en valeur ceux qui avaient témoigné des pratiques culturelles et à permettre à la population d'entrer en contact avec ces ressources ethnologiques.

D'autres activités se sont tenues au Musée Marius-Barbeau en Beauce, avec la Société historique de la Côte-Nord à Baie-Comeau et au Musée québécois de culture populaire à Trois-Rivières. Les équipes de ces régions ont organisé des "Soirées du patrimoine en apéro » qui ont rassemblé les artisans, les intervenants du milieu, les chercheurs universitaires, les partenaires de l'IREPI et la population locale. Par ailleurs, l'équipe de la Capitale-Nationale a réalisé une exposition muséale au Musée de Charlevoix, en collaboration avec des partenaires 
du milieu. Inaugurée le 17 décembre, l'exposition de photographies, qui s'accompagnait d'un guide audio rassemblant des extraits d'entrevues réalisées au cours de l'été 2006, a été un franc succès. L'exposition a également été présentée à la salle d'exposition du pavillon AlphonseDesjardins de l'Université Laval à l'automne 2007. Le Musée québécois de culture populaire à Trois-Rivières, partenaire de la Chaire, prépare pour 2008 une exposition internationale sur le patrimoine immatériel en collaboration avec le Musée de la vie wallonne à Liège.

\section{Activités de terrain en 2007}

Dans plusieurs régions visitées en 2006, les équipes sur le terrain avaient repéré plusieurs ressources potentielles. Riches de cette expérience précédente, les responsables de l'IREPI ont planifié le terrain 2007 en tenant compte des ressources du patrimoine immatériel déjà identifiées et de l'avancement de la collecte dans certaines MRC couvertes en 2006 afin de consolider les acquis et d'ouvrir de nouveaux chantiers en patrimoine immatériel. Après une semaine de formation sur le patrimoine immatériel et sur le matériel informatique et audiovisuel du Laboratoire d'enquête et d'entrevue multimédia (LEEM), deux étudiants au baccalauréat et six étudiants à la maîtrise se sont installés pendant onze semaines dans cinq régions du Québec : dans le Bas-SaintLaurent ; à la MRC de Kamouraska, St-Pascal ; au Musée de la mémoire vivante, à St-Jean-Port-Joli en Chaudière-Appalaches; au Musée de Charlevoix et au centre d'interprétation de la Côte de Beaupré pour la Capitale-Nationale; à Baie-Comeau et au Musée régional de la CôteNord à Sept-Îles, pour la Côte-Nord; et dans la région de Lanaudière (premier inventaire dans cette région du Québec). D'autre part, trois nouveaux enquêteurs ont été recrutés par la Chaire pour que l'inventaire des ressources ethnologiques du patrimoine immatériel soit représentatif à la fois des régions et des communautés, et afin d'assurer une bonne représentation des formes d'expression, en particulier des fêtes et des festivals qui se tiennent toute l'année.

L'inventorisation des données recueillies en 2007 a commencé en août. Le site Internet a été amélioré afin d'offrir à l'internaute une recherche par carte géographique. Cette nouvelle fonction permet de rechercher les ressources soit d'une région, soit d'une MRC, à l'aide de cartes cliquables. Le site s'est également enrichi de fiches descriptives rédigées à partir de données recueillies lors de projets-pilotes menés à l'extérieur du Canada sous la supervision de la Chaire. 


\section{Projets-pilotes}

Au cours l'année 2007, la Chaire de recherche en patrimoine ethnologique a collaboré à la mise en œuvre de projets-pilotes d'inventaire du patrimoine immatériel avec la Belgique et Haïti. Au printemps 2007, dans le cadre du projet de coopération Inventaire du patrimoine immatériel Québec/Wallonie-Bruxelles, Françoise Lempereur, chercheuse responsable du volet de l'inventaire du patrimoine immatériel en Belgique et maître de conférences à l'Université de Liège, a rencontré à l'Université Laval les chercheurs de la Chaire afin de se familiariser avec la méthodologie développée pour l'inventaire du patrimoine immatériel au Québec. En avril, un projet-pilote a été mené en Haïti afin d'évaluer la faisabilité d'un inventaire national. Les résultats ont été fort concluants. En mai, Richener Noël, Lewis Ampidu Clormeus, Kenrick Desmavar, Samuel Regulus, étudiants à la maîtrise en patrimoine à l'Université d'État d'Haïti, et le professeur Pierre-Louis Ricot sont venus parfaire leur formation auprès de l'équipe de l'inventaire des ressources ethnologiques du patrimoine immatériel de l’Université Laval.

\section{Diffusion de la recherche}

En septembre 2006, Laurier Turgeon et Martine Roberge ont présenté l'Inventaire des ressources ethnologiques du patrimoine immatériel (IREPI) à l'Université de Liège en Belgique, à la Direction du patrimoine de l'UNESCO à Paris, ainsi que dans le cadre du Colloque international sur le patrimoine immatériel à Florence. Ces présentations ont suscité plusieurs projets de collaboration. Ainsi, en mai 2007, la Chaire de recherche du Canada en patrimoine ethnologique a reçu une délégation belge dans le cadre d'un projet commun d'inventaire du patrimoine immatériel Québec-Belgique. En février 2007, le LEEM a reçu Frédérique Boura, conservatrice en chef du patrimoine de la Région Alsace et Sébastien Soubiran, chercheur à la Mission scientifique et technique de l'Université Louis Pasteur, Strasbourg. Puis en juin 2007, Patricia Heiniger, chercheure à l'Université de Pau a ainsi visité le LEEM et les Archives de folklore et d'ethnologie de l'Université Laval dans le but d'explorer la possibilité de mettre sur pied un programme de maîtrise axé sur la conservation du patrimoine et des archives audio-visuelles.

Durant l'hiver 2007, Laurier Turgeon a profité d'un séjour d'études en France pour faire valoir le projet d'inventaire du patrimoine 
immatériel au Québec dans plusieurs pays européens. Ainsi, en février 2007, une présentation a été faite au séminaire du LAHIC (École des hautes études en sciences sociales) dirigé par Daniel Fabre, intitulée «Le patrimoine immatériel et le rôle des inventaires. L'exemple de l'IREPI au Québec ». Une autre présentation s'est déroulée au Service de l'Inventaire, ministère de la Culture de la France, intitulée «Faire l'inventaire du patrimoine immatériel du Québec. Problématiques, méthodes et enjeux ». Ce séjour d'études a également donné lieu à plusieurs rencontres qui ont servi à mettre en valeur le projet de l'IREPI.

\section{Inventaire du patrimoine immatériel religieux}

La Chaire a été mandatée par le ministère québécois de la Culture, des Communications et de la Condition féminine pour réaliser un projetpilote de l'inventaire du patrimoine immatériel du Québec. Au cours de l'automne 2007, une équipe d'ethnologues rencontrera les porteurs de tradition de différentes confessions. Les enquêtes seront menées dans deux congrégations catholiques fondatrices (une féminine et une masculine) et quatre communautés représentant les minorités religieuses (anglicane, juive, orthodoxe, amérindienne) ainsi que dans deux paroisses (protestante et catholique). Les objectifs du projet-pilote sont de mesurer l'étendue et la nature du patrimoine immatériel religieux au Québec, de développer une méthodologie appropriée, des outils d'enquête et de classification efficaces, de procéder à une cueillette de données et à leur traitement en vue d'élaborer un inventaire informatisé. Ce projet-pilote servira de modèle à la mise en œuvre d'un inventaire national du patrimoine immatériel religieux québécois.

\section{L'Encyclopédie du patrimoine culturel de l'Amérique française}

Ce projet est né à l'initiative de Marcel Masse, président de la Société Héritage de Champlain. Dirigé par le titulaire de la Chaire de recherche du Canada en patrimoine et codirigé par Yves Bergeron, professeur à l'Université du Québec à Montréal, l'ensemble du projet est coordonné par Martin Fournier.

L'Encyclopédie du patrimoine culturel de l'Amérique française est un projet novateur qui présente le patrimoine francophone du Canada et de l'Amérique du Nord comme un phénomène pluriel et dynamique toujours en construction. Il traite des principaux éléments de ce patrimoine diversifié, sous toutes ses formes et dans toutes les régions 
du continent, pendant les quatre siècles de son histoire. Il inclut également le patrimoine français d'origine et les relations entre la France et l'Amérique française.

L'Encyclopédie décrit dans un premier temps l'état actuel du patrimoine, puis elle explique les parcours de construction et les démarches de mise en valeur des pratiques et des biens patrimoniaux retenus au cours de leur évolution, c'est-à-dire leur patrimonialisation. Cette approche convient bien au cas des francophones d'Amérique du Nord dont le patrimoine est marqué par des transformations et des emprunts significatifs, notamment aux cultures autochtones, britanniques et américaines avec lesquelles ils ont été successivement en contact. Ces adaptations confèrent au patrimoine de l'Amérique française son caractère propre et sa richesse.

Au total, l'Encyclopédie offrira une sélection de plus de 500 articles d'une longueur moyenne de 1000 à 2000 mots chacun. Chaque article sera complété par de nombreux documents textuels, sonores, visuels et audiovisuels. L'Encyclopédie sera dans un premier temps diffusée sur un site Internet bilingue (français/anglais) à compter du printemps 2008. Le projet sera réalisé dans son ensemble sur une période de cinq ans.

Célia Forget, Louise Saint-Pierre et Martin Fournier 